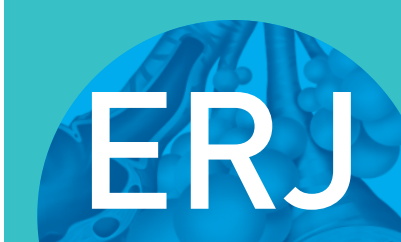

open research

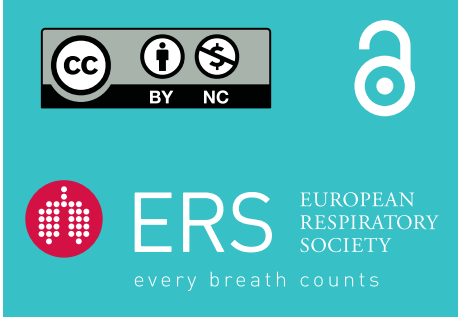

\section{Pre-COVID-19 lung function and other risk factors for severe COVID-19 in first responders}

\author{
To the Editor:
}

Starting 1 March 2020, New York City (NYC) suffered an intense coronavirus disease 2019 (COVID-19) outbreak caused by severe acute respiratory syndrome coronavirus 2 (SARS-CoV-2). By 31 May 2020, there were 203248 diagnosed patients, 52301 hospitalisations and 17756 confirmed deaths in NYC [1]. Cardiac arrests with resuscitation increased three-fold compared with the same time period in 2019 [2]. Despite proper personal protective equipment (PPE) and compliance with Centers for Disease Control guidelines [3], medical leave for suspected/confirmed COVID-19 in Fire Department of the City of New York (FDNY) responders was substantial, affecting $40.7 \%$ of emergency medical service providers (EMS) and 34.5\% of firefighters [4]. We examined risk factors for COVID-19 diagnosis and severe COVID-19 disease (hospitalisation or death) in FDNY responders.

Our study population included FDNY firefighters and EMS who were actively employed on 1 March 2020 and had received $\geqslant 2$ pulmonary function tests (PFTs) between their hire date and 29 February 2020. COVID-19 diagnoses, hospitalisations and deaths between 1 March 2020 and 31 May 2020 were obtained from the FDNY electronic medical record. Responders who had a positive nucleic-acid COVID-19 test had confirmed COVID-19, and those with reported symptoms consistent with COVID-19 but not tested were classified as suspected COVID-19. Additionally, those who had both a confirmed diagnosis and a COVID-19-related hospitalisation or death were classified as severe COVID-19. Multivariate logistic regression assessed whether class of employment (firefighter or EMS), longitudinal rate of forced expiratory volume in $1 \mathrm{~s}\left(\mathrm{FEV}_{1}\right)$ decline/year between participants' first PFT and 1 March 2020, smoking history, age, sex, race, body mass index (BMI), blood glucose and high-density lipoprotein cholesterol levels were associated with either severe COVID-19, confirmed COVID-19 or suspected COVID-19. Analyses were performed using SAS, version 9.4 (SAS Institute, Cary, NC, USA). We followed STROBE (Strengthening the Reporting of Observational Studies in Epidemiology) reporting guidelines. The Montefiore Medical Center/Albert Einstein College of Medicine Institutional Review Board approved this study (\#2020-11983).

Of 15670 FDNY responders employed on 1 March 2020, the study population $(n=14290)$ comprised 3501 EMS and 10789 firefighters. Participants had a mean age of $40.4 \pm 9.2$ years, a mean BMI of $29.8 \pm 4.5 \mathrm{~kg} \cdot \mathrm{m}^{-2}$, and were $8.0 \%$ female, $67.8 \%$ white, $11.2 \%$ black, $16.6 \%$ Hispanic and $4.4 \%$ other races/ethnicities. Sixty-eight per cent were never-smokers, $25.1 \%$ were former-smokers, $4.1 \%$ current-smokers and $2.9 \%$ had missing smoking status. The mean \pm SD number of $\mathrm{FEV}_{1}$ measurements used in calculating $\mathrm{FEV}_{1}$ decline was 10.9 \pm 4.9 , and the mean duration was $11.9 \pm 6.1$ years. From 1 March 2020 to 31 May 2020, 9115 responders had no COVID-19 diagnosis, 5175 (362.1 out of 1000) were confirmed or suspected COVID-19 cases, and 62 (4.4 out of 1000) were hospitalised. Three participants died in hospital and one died at home ( 0.3 out of 1000). Responders aged 18-44 had a lower rate of COVID-19-related hospitalisations (3.0 out of 1000) and experienced no COVID-19-related deaths, despite having an elevated infection rate (405.3 out of 1000). EMS had more cases of severe COVID-19 than firefighters (42 out of 3501 (1.2\%) versus 21 out of 10789 (0.2\%);

@ERSpublications

Risk factors for \#COVID19 infection and severe disease (hospitalisation or death) in NYC first responders: greater pre-pandemic rate of $\mathrm{FEV}_{1}$ decline is associated with severe COVID-19, as is emergency medical service work versus firefighting https://bit.ly/3nZPuZY

Cite this article as: Weiden MD, Zeig-Owens R, Singh A, et al. Pre-COVID-19 lung function and other risk factors for severe COVID-19 in first responders. ERJ Open Res 2021; 7: 00610-2020 [https://doi.org/10.1183/23120541.00610-2020].

Copyright @ERS 2021. This article is open access and distributed under the terms of the Creative Commons Attribution NonCommercial Licence 4.0. 
Fisher's exact p-value <0.001). EMS were slightly younger than firefighters (mean \pm SD: $38.7 \pm 10.2$ versus $41.0 \pm 8.8$ years) and had a larger proportion of females $(29.3 \%$ versus $1.1 \%)$ and non-white individuals (60.7\% versus $22.9 \%$ ). After adjustment for confounders, multivariate logistic regression showed that EMS had a 4.23-fold increased odds of severe disease (95\% CI 2.20-8.15) when compared with firefighters (table 1). EMS also had a modest increase in odds of confirmed and a non-significant increase in odds of suspected COVID-19 (table 1).

A greater rate of $\mathrm{FEV}_{1}$ decline prior to 1 March 2020 was associated with severe COVID-19 but was not associated with confirmed or suspected COVID-19 infection. Compared with never-smokers, ever-smokers did not have increased odds of severe or confirmed COVID-19. Higher age, BMI, blood glucose level and non-white race were independent risk factors for severe COVID-19.

Overall, between 1 March 2020 and 31 May 2020 we observed that the COVID-19 infection rate among FDNY responders was 362 out of 1000 - 15 times the NYC rate (24 out of 1000) [1]. EMS had over a four-fold increased risk of severe COVID-19 and a 26\% increased risk of confirmed COVID-19 disease when compared with firefighters. It is likely that their higher-intensity COVID-19 exposures contributed to the increased risk and severity of COVID-19 illness. Although both FDNY firefighters and EMS responded to the pandemic-related surge in 9-1-1 emergency medical calls and followed the same PPE protocols [4], EMS had greater COVID-19 exposure than firefighters due to greater COVID-19-related call volume and being solely responsible for patient transport, nebulisation of bronchodilators and intubation.

Despite having a higher COVID-19 case rate than NYC, FDNY responders had a lower rate of severe COVID-19 than the city as a whole (4.1 out of 1000 versus 6.2 out of 1000 hospitalisations, and 0.3 out of 1000 versus 2.1 out of 1000 deaths) [1]. This may be due to the healthy worker effect, i.e. lower prevalence of comorbidities in FDNY responders than the general NYC population. The lower hospitalisation and death rates observed in this study may be a result of differences in the age distributions of the FDNY cohort and the general NYC population. The COVID-19-related hospitalisation rate in FDNY responders aged 18-44 was similar to that of NYC residents in the same age group (3.0 out of 1000 versus 2.8 out of 1000), though the infection rate was over 15 -fold higher (405.3 out of 1000 versus 25.5 out of 1000) [1].

As seen in other healthcare worker cohorts, higher age was associated with lower risk of COVID-19 infection [5]. This may be due to age-related changes in behaviour. Alternatively, since immunity to coronaviruses increases with age [6], cross-reactive cellular immunity to other coronaviruses may provide protection from contracting SARS-Cov-2 [7].

The FDNY longitudinal medical monitoring programme enabled assessment of $\mathrm{FEV}_{1}$ decline rate prior to the COVID-19 pandemic as a potential risk factor. We previously found that greater $\mathrm{FEV}_{1}$ decline rates were associated with asthma and COPD in World Trade Center-exposed FDNY responders [8]. In our current study, we observed greater $\mathrm{FEV}_{1}$ decline rates to be associated with severe COVID-19 disease. Further investigation of parameters of lung health associated with severe COVID-19 are needed in other longitudinal cohorts. Interestingly, we observed confirmed COVID-19 diagnosis was not associated with smoking and that ever-smoking was less prevalent in severe COVID-19 disease, a surprising finding as

TABLE 1 Multivariate logistic regression model predicting coronavirus disease 2019 (COVID-19)-related outcomes

\begin{tabular}{|c|c|c|c|c|c|c|}
\hline \multirow[t]{2}{*}{ Variables } & \multicolumn{2}{|c|}{$\begin{array}{l}\text { Hospitalisation/death versus } \\
\text { no COVID-19 diagnosis }\end{array}$} & \multicolumn{2}{|c|}{$\begin{array}{l}\text { Confirmed COVID-19 versus } \\
\text { no COVID-19 diagnosis }\end{array}$} & \multicolumn{2}{|c|}{$\begin{array}{l}\text { Suspected COVID-19 versus } \\
\text { no COVID-19 diagnosis }\end{array}$} \\
\hline & OR $(95 \% \mathrm{CI})$ & p-value & OR $(95 \% \mathrm{CI})$ & p-value & OR $(95 \% \mathrm{CI})$ & p-value \\
\hline Subjects $n$ & 63 & & 1569 & & 3543 & \\
\hline EMS versus firefighter & $4.23(2.20-8.15)$ & $<0.001$ & $1.28(1.10-1.49)$ & 0.001 & $1.07(0.96-1.20)$ & 0.228 \\
\hline $\mathrm{FEV}_{1}$ decline per $100 \mathrm{~mL} \cdot$ year $^{-1}$ & $1.70(1.12-2.59)$ & 0.012 & $0.93(0.81-1.06)$ & 0.265 & $1.01(0.92-1.11)$ & 0.803 \\
\hline Ever-smoker & $0.46(0.25-0.86)$ & 0.014 & $0.99(0.88-1.12)$ & 0.920 & $1.14(1.04-1.24)$ & 0.005 \\
\hline Non-white race & $2.46(1.34-4.51)$ & 0.004 & $1.21(1.06-1.38)$ & 0.004 & $1.24(1.13-1.37)$ & $<0.001$ \\
\hline BMI & $1.09(1.05-1.13)$ & $<0.001$ & $1.02(1.00-1.03)$ & 0.029 & $1.00(0.99-1.01)$ & 0.665 \\
\hline Blood glucose $\mathrm{mmol} \cdot \mathrm{L}^{-1}$ & $1.13(1.02-1.25)$ & 0.015 & $0.95(0.89-1.01)$ & 0.093 & $1.01(0.97-1.05)$ & 0.626 \\
\hline HDL cholesterol mmol. $\mathrm{L}^{-1}$ & $0.38(0.15-0.97)$ & 0.042 & $1.11(0.94-1.30)$ & 0.222 & $0.95(0.84-1.07)$ & 0.359 \\
\hline
\end{tabular}


smoking increases expression of the SARS-Cov-2 receptor in human lung epithelium $[9,10]$. However, the impact of smoking on COVID-19 is complex, and while our findings are consistent with ecological epidemiology studies where smoking was less prevalent in hospitalised patients, further studies are needed before reaching any conclusion [11-14]. Lastly, similar to other reports, we found that non-white race was strongly associated with COVID-19-related hospitalisation [15].

This study has limitations. The data are derived from a single occupational cohort, limiting its generalizability. As with all observational studies, there may be residual confounding. Since molecular testing for COVID-19 was limited early on in the NYC outbreak, a majority of FDNY responders who had symptoms of COVID-19 did not have a confirmed COVID-19 diagnosis via a positive nucleic acid test. The similarity in risk factors for suspected and confirmed COVID-19 in this cohort lends support to the accuracy of symptom screening for COVID-19 diagnosis in high prevalence settings; however, respiratory symptoms related to smoking or seasonal influenza may reduce the specificity of suspected COVID-19 diagnosis. These limitations are balanced by the strength of a closed cohort with extensive information from prior monitoring exams and with paid medical leave, reducing the likelihood of selection bias as well as ascertainment biases.

In summary, our study emphasises the elevated risk of disease present in FDNY responders during the COVID-19 pandemic, particularly EMS and those with greater pre-pandemic lung function decline. To protect healthcare workers, including pre-hospital responders, requirements of proper PPE use, environmental controls, influenza vaccination (to minimise additional medical leave, mischaracterisation and potential additional comorbidity) and COVID-19 vaccination, when available, are all critical. Without these measures, healthcare systems will be unable to maintain surge capacity during this pandemic.

Michael D. Weiden ${ }^{1,2}$, Rachel Zeig-Owens $\oplus^{1,3,4}$, Ankura Singh ${ }^{1,3}$, Theresa Schwartz ${ }^{1,3}$, Yang Liu ${ }^{1,3}$, Brandon Vaeth ${ }^{1,3}$, Anna Nolan ${ }^{1,2}$, Krystal L. Cleven ${ }^{3}$, Karen Hurwitz ${ }^{1}$, Shenecia Beecher ${ }^{1}$ and David J. Prezant ${ }^{1,3,5}$

${ }^{1}$ The Bureau of Health Services and the FDNY World Trade Center Health Program, Fire Dept of the City of New York, Brooklyn, NY, USA. ${ }^{2}$ Pulmonary, Critical Care and Sleep Medicine Division, Depts of Medicine and Environmental Medicine, New York University School of Medicine, New York, NY, USA. ${ }^{3}$ Pulmonary Medicine Division, Dept of Medicine, Montefiore Medical Center and Albert Einstein College of Medicine, Bronx, NY, USA. ${ }^{4}$ Division of Epidemiology, Dept of Epidemiology and Population Health, Albert Einstein College of Medicine, Bronx, NY, USA. ${ }^{5}$ Office of Medical Affairs, Fire Dept of the City of New York, Brooklyn, NY, USA.

Correspondence: David Prezant, 9 MetroTech Center, Brooklyn, NY 11201, USA. E-mail: David.Prezant@ fdny.nyc.gov

Received: 24 Aug 2020 | Accepted after revision: 6 Oct 2020

Author contributions: M.D. Weiden, D.J. Prezant and R. Zeig-Owens designed the study. M.D. Weiden, A. Singh, T. Schwartz, Y. Liu, B. Vaeth, A. Nolan, K. Hurwitz, S. Beecher and D.J. Prezant performed data curation. M.D. Weiden, R. Zeig-Owens and A. Singh performed data analyses. M.D. Weiden prepared the first draft of the manuscript. M.D. Weiden, R. Zeig-Owens, A. Singh, A. Nolan, K.L. Cleven and D.J. Prezant critically revised the manuscript. All authors have read and approved the manuscript. D.J. Prezant takes the responsibility for the integrity of the data.

Conflict of interest: M.D. Weiden has nothing to disclose. R. Zeig-Owens reports grants from NIOSH outside the submitted work. A. Singh has nothing to disclose. T. Schwartz has nothing to disclose. Y. Liu has nothing to disclose. B. Vaeth has nothing to disclose. A. Nolan has nothing to disclose. K.L. Cleven has nothing to disclose. K. Hurwitz has nothing to disclose. S. Beecher has nothing to disclose. D.J. Prezant has nothing to disclose.

\section{References}

1 New York City Department of Health and Mental Hygiene. COVID-19: Data - coronavirus-data/case-hospdeath.csv. NYC Department of Health and Mental Hygiene, 2020. https:/github.com/nychealth/coronavirus-data/ blob/master/case-hosp-death.csv.

2 Lai $\mathrm{PH}$, Lancet EA, Weiden MD, et al. Characteristics associated with out-of-hospital cardiac arrests and resuscitations during the novel coronavirus disease 2019 pandemic in New York City. JAMA Cardiol 2020; 5: $1154-1163$.

3 Centers for Disease Control and Prevention. Interim Guidance for Emergency Medical Services (EMS) Systems and 911 Public Safety Answering Points (PSAPs) for COVID-19 in the United States 2020. www.cdc.gov/ coronavirus/2019-ncov/hcp/infection-control-recommendations.html.

4 Prezant DJ, Zeig-Owens R, Schwartz T, et al. Medical leave associated with COVID-19 among emergency medical system responders and firefighters in New York City. JAMA Netw Open 2020; 3: e2016094.

5 Houlihan CF, Vora N, Byrne T, et al. Pandemic peak SARS-CoV-2 infection and seroconversion rates in London frontline health-care workers. Lancet 2020; 396: e6-e7. 
6 Gorse GJ, Donovan MM, Patel GB. Antibodies to coronaviruses are higher in older compared with younger adults and binding antibodies are more sensitive than neutralizing antibodies in identifying coronavirus-associated illnesses. J Med Virol 2020; 92: 512-517.

7 Grifoni A, Weiskopf D, Ramirez SI, et al. Targets of T cell responses to SARS-CoV-2 coronavirus in humans with COVID-19 disease and unexposed individuals. Cell 2020; 181: 1489-501.

8 Singh A, Liu C, Putman B, et al. Predictors of asthma/COPD overlap in FDNY firefighters with World Trade Center dust exposure: a longitudinal study. Chest 2018; 154: 1301-1310.

9 Cai G, Bossé Y, Xiao F, et al. Tobacco smoking increases the lung gene expression of ACE2, the receptor of SARS-CoV-2. Am J Respir Crit Care Med 2020; 201: 1557-1559.

10 Leung JM, Yang CX, Tam A, et al. ACE-2 expression in the small airway epithelia of smokers and COPD patients: implications for COVID-19. Eur Respir J 2020; 55: 2000688.

11 Polverino F. Cigarette smoking and COVID-19: a complex interaction. Am J Respir Crit Care Med 2020; 202: 471-472.

12 Tsigaris P, Teixeira da Silva JA. Smoking prevalence and COVID-19 in Europe. Nicotine Tob Res 2020; 22: 1646-1649.

13 Rossato M, Russo L, Mazzocut S, et al. Current smoking is not associated with COVID-19. Eur Respir J 2020; 55 2001290.

14 Russo P, Bonassi S, Giacconi R, et al. COVID-19 and smoking: is nicotine the hidden link? Eur Respir J 2020; 55 2001116.

15 Karaca-Mandic P, Georgiou A, Sen S. Assessment of COVID-19 hospitalizations by race/ethnicity in 12 states. JAMA Intern Med 2021; 181: 16-22. 